\title{
ANÁLISIS DEL FLAMENCO COMO RECURSO TURÍSTICO EN ANDALUCÍA
}

\author{
$M^{a}$ Genoveva Millán Vázquez de la Torre* \\ Salud Millán Lara** \\ Juan Manuel Arjona Fuentes* \\ Universidad Loyola Andalucía
}

\section{RESUMEN}

El flamenco es un arte que nace en Andalucía resultado de una fusión de culturas que convivieron durante siglos en esta tierra. La fuerza del flamenco hace que se haya convertido en marca de identificación de Andalucía y de España, lo que se traduce en una demanda exterior y una afluencia de turistas conocedores del flamenco o con curiosidad por el mismo. El turismo cultural del flamenco, tiene una importante repercusión económica sobre el sector del espectáculo, de la formación, hostelería y otras industrias asociadas, como la de complementos flamencos. El flamenco como marca debe tener un apoyo público y privado andaluz que potencie su desarrollo y favorezca la afluencia de turismo cultural.

Palabras clave: Flamenco, Turismo del flamenco, Turismo cultural, DAFO, Business Model Canvas.

\section{Analysis of the flamenco as a tourist resource in Andalusia}

\section{ABSTRACT}

Flamenco is an art born in Andalusia a saresult of a fusion of the cultures that coexisted in this land for centuries. The strength of flamenco has made it an identifying emblem of Andalusia and of Spain, which translates into foreign acclaim and an influx of tourists

Recibido: 6 de marzo de 2015

Devuelto para su revisión: 23 de junio de 2015

Aceptado: 24 de septiembre de 2015

* Departamento de Métodos Cuantitativos. Universidad Loyola Andalucía. C/ Escritor Castilla Aguayo, $\mathrm{n}^{\circ}$ 4,14004 CÓRDOBA (España).E-mail: gmillan@uloyola.es, jmarjona@uloyola.es

** Departamento de Economía Financiera y Contabilidad. Universidad Loyola Andalucía. C/ Escritor Castilla Aguayo, nº 4, 14004 CÓRDOBA (España). E-mail: smillan@uloyola.es 
knowledgeable about flamenco or intrigued by it. Cultural tourism with respect to flamenco has a significant economic impact on the entertainment, training and catering sectors as well as other related industries, like that of flamenco accessories. Flamenco as a brand should have public and private Andalusian support to enhance its development and encourage the influx of cultural tourism.

Keywords: Flamenco; Flamenco tourism; Cultural tourism; SWOT; Business Model Canvas.

\section{INTRODUCCIÓN}

El tránsito de la sociedad moderna a la sociedad posmoderna está contribuyendo a transformar la demanda turística. Actualmente se percibe en la sociedad una búsqueda de equilibrio entre el hombre y su entorno natural y sociocultural. Este proceso tiene reflejo en el turismo que se encuentra en una etapa de madurez, marcada por el cuestionamiento del modelo tradicional de sol y playa, la aparición de nuevas motivaciones en la demanda turística y la especialización de la oferta turística. Así, el turismo se adapta a las nuevas demandas de sus consumidores, cada vez más exigentes en cuanto a la calidad y variedad de los servicios y con una amplia variedad de motivaciones para viajar. Este modelo de turismo alternativo acentúa el interés por los espacios mejor conservados y por otra clase de atractivos más específicos.

Nos interesa centrarnos en la modalidad de turismo cultural o del patrimonio, es decir, aquél que se desarrolla en lugares con historia como principal tema de atracción y que proporciona al turista una experiencia que supone una ampliación de sus conocimientos culturales. Recordemos que el término cultura abarca multiplicidad de manifestaciones, a) lo que piensan las personas (actitudes, creencias, ideas o valores), b) lo que hacen (pautas de comportamiento normativo o modos de vida) y lo que fabrican (obras de arte o productos). Pero ¿Qué se entiende por patrimonio?, El patrimonio es un concepto diverso, poliédrico y con diversas manifestaciones: cultural, artística, arqueológica, histórica, etnológica, documental, militar, natural, escénica o de cualquier otra que pudiese surgir. De ahí que se hable de patrimonio cultural, de patrimonio artístico, de patrimonio arqueológico, etc. La visión sobre el patrimonio se entiende como flexible y dinámica, ya que identificamos en ella al conjunto de elementos y prácticas sociales a través de las cuáles un colectivo pretende reconocerse y representarse.

Por tanto podemos distinguir entre patrimonio:

a) Tangible: Referido a los bienes materiales que se pueden percibir de manera precisa.

- Inmueble: edificios, iglesias, monumentos, castillos, fábricas, talleres, minas, almacenes, colonias obreras, etc...

- Mueble: Archivos documentales, fotografías, máquinas, herramientas, etc...

b) Intangible: Referido a todo aquello no material que rodea a la cultura, tradiciones, costumbres, formas de vida, saber-hacer, cante, baile, etc... 
El concepto de patrimonio ha estado siempre muy sesgado hacia los valores que se podrían denominar «histórico-artísticos», cuando en realidad abarca mucho más entre ellas a la cultura.

En el pasado el interés se centró en castillos, catedrales, iglesias o museos, habiendo sido potenciados como reclamo turístico. Un concepto moderno de patrimonio cultural incluye no solo los monumentos y manifestaciones del pasado (sitios y objetos arqueológicos, arquitectura colonial e histórica, o documentos y obras de arte), sino también lo que se llama patrimonio vivo, incluyendo, por tanto el patrimonio derivado de la cultura y formas de vida.

La Constitución española en su art. 46 consagra la conservación y enriquecimiento del patrimonio histórico cultural y artístico como una de las funciones que deben asumir los poderes públicos.

La Unión Europea tiene un programa de apoyo a proyectos piloto comunitarios en materia de Conservación del patrimonio.

El Estatuto de Autonomía para Andalucía establece en el artículo 10.3 uno de los objetivos básicos de la Comunidad Autónoma: «afianzar la conciencia de identidad andaluza, a través de la investigación, difusión y conocimiento de los valores históricos, culturales y lingüísticos del pueblo andaluz en toda su riqueza y variedad», atribuyendo el artículo 68 de la misma norma legal la competencia exclusiva de la Comunidad Autónoma en materia de Patrimonio Histórico, Artístico, Monumental, Arqueológico y Científico.

El turismo cultural, basado en el patrimonio de una comunidad, es una forma de turismo alternativo que trata de aprovechar el potencial interés cultural que los visitantes puedan tener sobre el patrimonio tangible e intangible del fenómeno de la industrialización. Podemos clasificar al turismo cultural en:

- Turismo arquitectónico: el objetivo es visitar y conocer las obras arquitectónicas del lugar, edificios, obras públicas, monumentos, etc. (Trotiño y Trotiño, 2009)

- Turismo gastronómico: el cual se realiza para conocer el proceso productivo de productos típicos de una zona geográfica (vino, aceite, etc.) y degustación de los mismos.

- Turismo de exposición: la finalidad del viaje es visitar una exposición cultural, pictórica, productos de artesanía, etc...

- Turismo religioso: Es aquél que se realiza por motivos espirituales con el fin último de visitar un lugar religioso.

- Turismo industrial: En este tipo de turismo se visitan empresas que están en desuso (minas) o en activo para conocer el proceso productivo.

- Turismo de compras: Es el que se realiza en una determinada zona, cuyo objetivo es la compra de productos, que no tienen por qué ser típicos del lugar sino que pueden ser de otras regiones o países, con la ventaja de ser más baratos para el consumidor final, por ser ciudades puerto franco al no pagar determinados impuestos (Ceuta, Gran Canarias).

- Turismo de idiomas: El objetivo de este tipo de turismo es aprender un idioma y su cultura. 
- Turismo étnico folclore: El turista desea conocer el folklore y tradiciones festivas del entorno que visita, siendo este tipo de turismo el que analizaremos en esta investigación.

El Turismo Cultural, por tanto juega un papel muy importante para dar a conocer, preservar y disfrutar el patrimonio cultural y turístico de un país (Molinar, 2006). De ahí la importancia de este estudio que pretende analizar la oferta de turismo cultural relacionado con el flamenco declarado Patrimonio Inmaterial de la Humanidad por la Unesco (2010), ya que bien desarrollado este producto turístico puede generar el desarrollo económico y social de la zona donde se realiza la actividad, puede incrementar el empleo en esta época de crisis económica, como indica Toselli (2003) el turismo cultural puede ser un positivo instrumento de desarrollo local y regional.

\section{EL FLAMENCO Y SU EVOLUCIÓN}

El flamenco es una expresión musical que transmite sentimientos, de amor, de desamor, de alegrías y penas, de lucha, de reproche, de reivindicación... es una expresión musical que nace del pueblo, por tanto forma parte de su cultura.

Sise indaga en la Región de Andalucía, nos encontramos con una encrucijada de culturas, una tierra en la que durante siglos convivió la cultura cristiana, musulmana, judía y gitana. Decir que el origen del flamenco procede del pueblo gitano es arriesgado, máxime teniendo en cuenta que los gitanos de otros países no desarrollan esta expresión musical. Si se afirma que el flamenco es el resultado de una fusión cultural en tierras andaluzas se puede considera que no está lejos de la realidad (Cruces, 2002).

Los trabajadores del campo, de la sierra o de la campiña, los del mar, los mineros, los herreros... entonaban sus cantes y expresaban sus emociones en sus bailes. Normalmente cada zona geográfica ofrece una expresión artística que la identifica. Si recorremos lugares de España cada uno muestra unas particularidades dignas de admiración: en Galicia la Muñeira, en Cataluña la Sardana, en Zaragoza la Jota... y así se podría seguir por todo el territorio español, pero todo esto se identifica con el concepto folklore, es decir, cantes y bailes que muestran la cultura de una zona geográfica pero que difícilmente transcienden sus fronteras.

El folklore andaluz traspasa las fronteras andaluzas y las fronteras españolas dejando de ser folklore para ser clasificado dentro de la categoría de arte, «el arte flamenco». El flamenco aunque tiene simientes en el folklore andaluz, éste evoluciona exigiendo a sus intérpretes unas cualidades interpretativas y físicas no comunes, que distinguen a sus artistas y les permiten una comunicación intensa (Torres, 2014); es lo que algunos llaman el «duende del flamenco». (Agenda de la Empresa Andaluza, 2014).

El arte flamenco se expresa en tres modalidades: cante, baile y guitarra. El cante normalmente necesita de la guitarra y el baile normalmente necesita del cante y de la guitarra. Los espectáculos de flamenco suelen ofrecer muestras de las tres vertientes aunque no es extraño ver por el mundo espectáculos centrados en cada una de ellas (Gómez, 2003). 
La música que nace del pueblo no nace como espectáculo sino como una necesidad de expresar sentimientos y estados de ánimos. Si se analizan algunos estilos flamencos, se observa como muestran su origen desde la actividad que los genera, por ejemplo: «la serrana» cante y baile procedente de la sierra y vinculado con los bandoleros; los cantes de «trilla» de los trabajadores de la campiña; «el taranto o las mineras» de las minas, «el martinete» de las herrerías o fraguas (Cáceres y Campo, 2013), y así se podría seguir haciendo referencias.

Lo que está claro es que el arte flamenco no surge con el propósito de generar una oferta como medio de vida y mucho menos para generar una atracción de visitantes a la zona. Es decir, el concepto turismo no cabe en estos orígenes.

Hay que trasladarse al siglo XVIII para hablar de los primeros artistas del flamenco pero también es cierto que se trataba de artistas cuyas actuaciones sólo se limitaban a participar en fiestas particulares de las que se tienen pocas referencias (Vergillos, 2002).

En el siglo XIX, en su segunda mitad, surgen los «cafés cantantes», locales en los que actuaban artistas profesionales de cante, baile y guitarra. Estos locales sacaron el flamenco de la privacidad del sector minoritario en busca de una difusión de masas.

\section{Figura 1 \\ CAFÉ CANTANTE DEL SIGLO XIX}

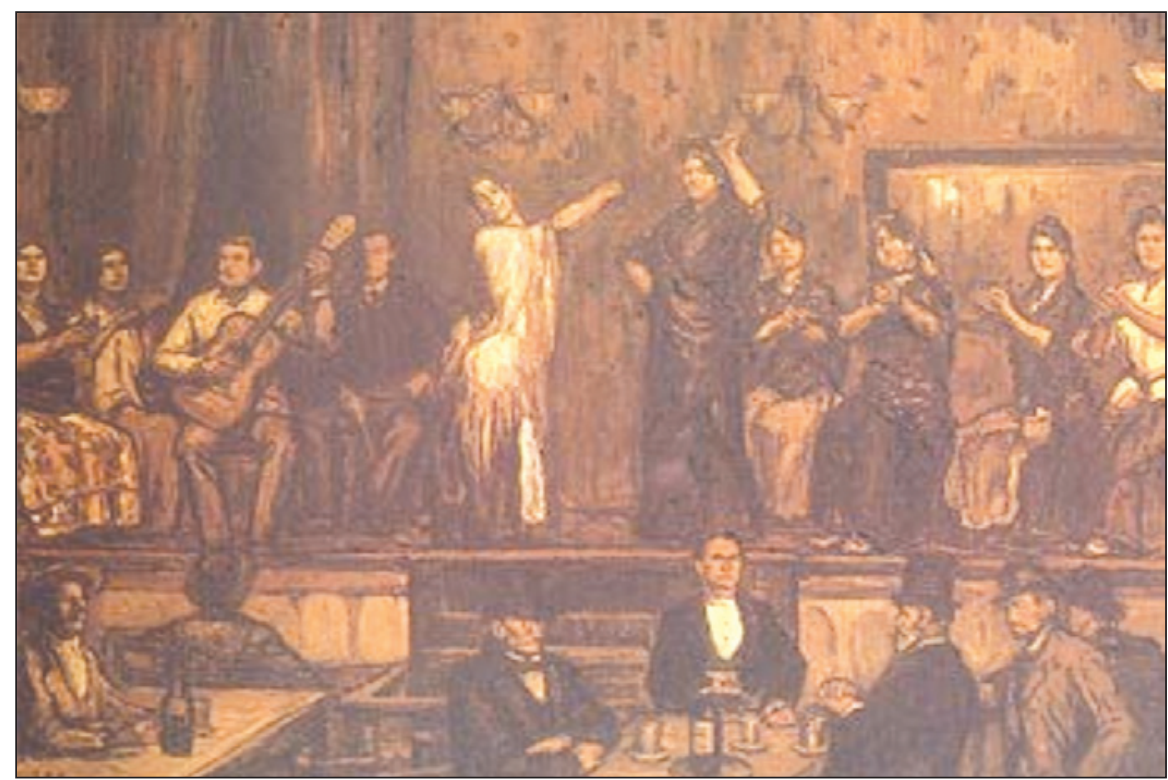

Fuente: https://antoniocamel.wordpress.com/2012/10/14/cafe-cantante/

Por primera vez, los artistas podían dedicarse en exclusiva a su arte sin tener que compaginarlo con otras profesiones para poder sobrevivir y se pueden encontrar empresarios centrados en la oferta del arte flamenco. 
A primeros del siglo $\mathrm{XX}$, los flamencos traspasan fronteras.

El flamenco llega a París, el arte flamenco, el arte andaluz interesa en la ciudad de la cultura europea. Al mismo tiempo en Cataluña surge una corriente en contra de los cafés cantantes, pero el nacionalismo catalán no pudo frente al éxito del flamenco (Holguín, 2013).

Es curioso como también a primeros del siglo XX surgió una corriente para devolver el flamenco al pueblo abandonando la profesionalización del mismo, encabezada por Manuel de Falla y Federico García Lorca. En esta línea, en 1922 en Granada, se celebró el primer concurso de cante en el que sólo podían participar aficionados desconocidos. El concurso más que servir para abandonar la profesionalización del flamenco sirvió para abrir las puertas a nuevos profesionales, tales como Manolo Caracol que con trece años recibió del concurso una mención especial y, llegó a ser uno de los grandes profesionales del flamenco.

Figura 2

CARTEL DEL PRIMER CONCURSO

DE CANTE FLAMENCO EN GRANADA (1922)

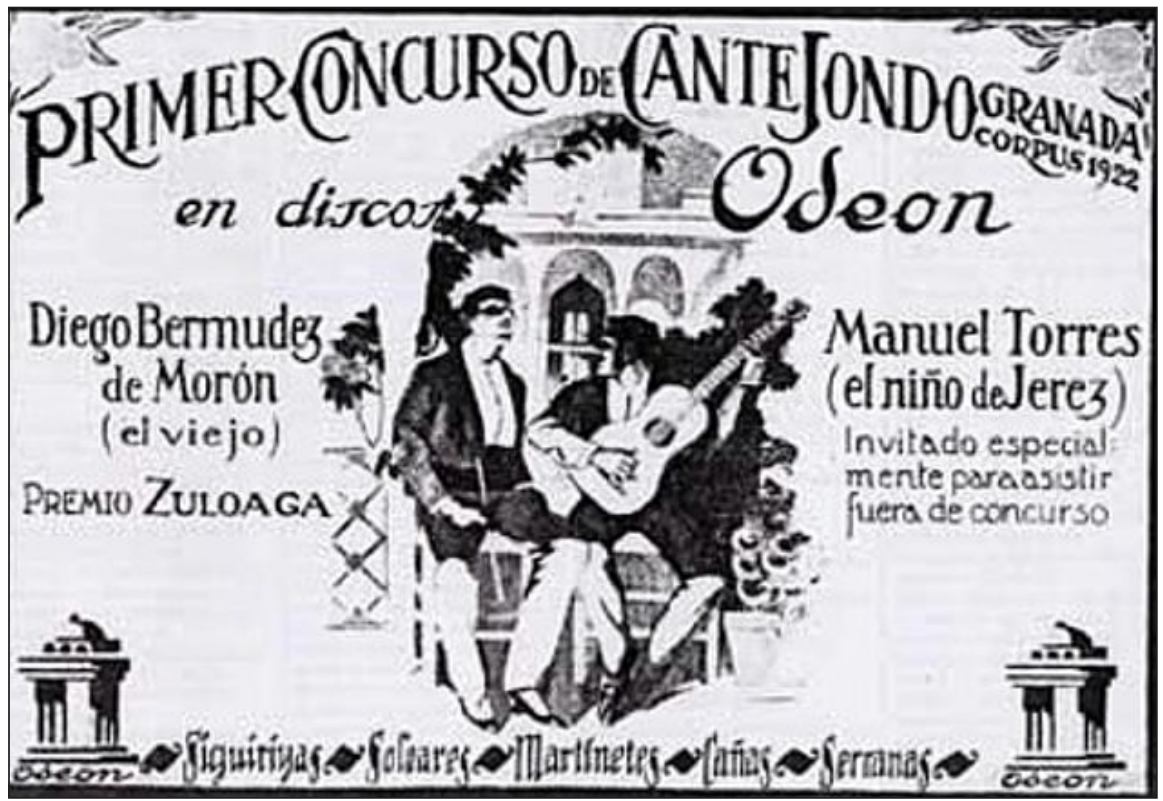

Fuente: http://nerva.mforos.com/905716/8093945-aficionados-al-cante-flamenco

Este concurso fue retomado en 1956 en Córdoba con la denominación de Concurso Nacional de Cante Jondo, que cada tres años ha venido celebrándose cambiándole el nombre a partir de la $4^{\text {a }}$ edición en 1965 a Concurso Nacional de Arte Flamenco ${ }^{1}$. El Nacional de

1 http://www.nacionaldearteflamenco.es/ 


\section{Figura 3 \\ CARTEL DEL CONCURSO NACIONAL DE ARTE FLAMENCO DE CÓRDOBA (1965)}

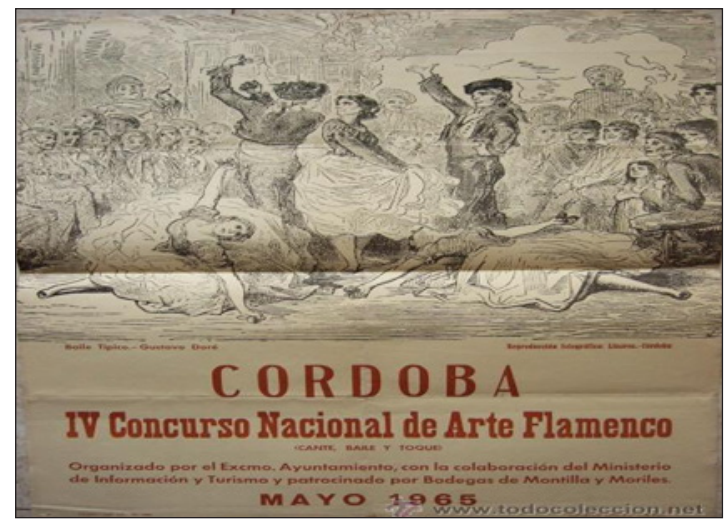

Fuente:http://www.todocoleccion.net/cartel-espectaculos/cartel-iv-festival-concurso-nacional-arte-flamencocordoba-1965 x36828200\#sobre_el_lote

\section{Figura 4}

CARTEL DE OPERA FLAMENCA (1940)

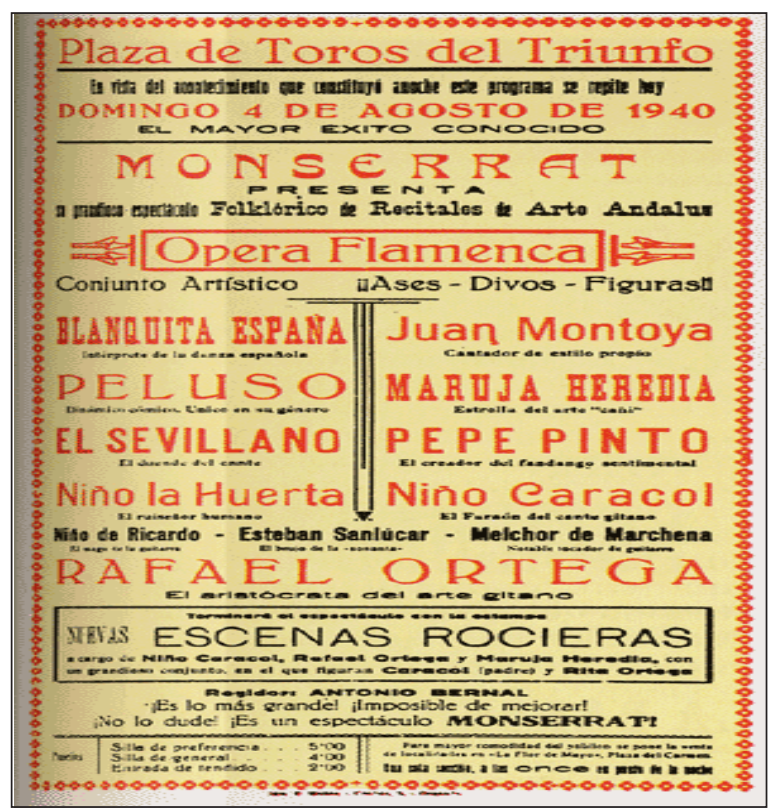

Fuente: https://albherto.wordpress.com/2010/04/03/opera-flamenca/ 
Córdoba pretendía rescatar y conservar la pureza del flamenco pero manteniendo la profesionalidad del mismo. De hecho para los ganadores, el premio nacional era como una graduación en flamenco que le permitía entrar en los circuitos de grandes festivales y espectáculos.

Este objetivo se mantiene hasta nuestros días.

Ciertamente en la primera mitad del siglo XX, en la capital de España empiezan a proliferar los cafés cantantes y los espectáculos de flamenco en los teatros con la denominación de «Ópera Flamenca». Esta denominación se debe en gran medida a que en 1926 los cafés cantantes y otros espectáculos tenían que tributar con un $10 \%$ mientras que los conciertos instrumentales y la ópera lo hacían sólo con un 7\%². El flamenco estaba dando sus frutos económicos y había que optimizarlos.

Como respuesta a la Ópera Flamenca que permitía y apoyaba principalmente los cantes fáciles de entender por el público general, además del Concurso Nacional de Arte Flamenco, surge la Cátedra de Flamencología de Jerez de la Frontera, el concepto de peñas flamencas y nace la época de los festivales flamencos. Lugares y eventos generadores de recursos para los profesionales del flamenco que luchaban por mantener la esencia del mismo (Gamboa, 2005).

Actualmente las fuentes de flamenco se sitúan tanto en el sector público como en el privado.

Desde el sector público se pueden destacar:

- Eventos patrocinados e impulsados por ayuntamientos de capitales andaluzas. Esta oferta suele ir acompañada de una importante difusión que sólo en algunos casos es capaz de atraer al turismo de masas.

- Organismos creados por la Junta de Andalucía como el Instituto Andaluz del Flamenco, perteneciente a la Consejería de Cultura, Educación y Deporte que desde 2005 presenta como objetivo la conservación y recuperación del arte flamenco así como su difusión y promoción. El instituto apuesta por el desarrollo nacional e internacional, por la formación en flamenco en los colegios y por el apoyo al movimiento asociativo que existe en torno al flamenco (Carrasco, 2014).

El hecho de que en 2010 se declarase al flamenco Patrimonio Cultural Inmaterial de la Humanidad por parte de la UNESCO, ha representado para las instituciones públicas un refuerzo en su compromiso de la defensa de este arte como marca de identificación (Cruces, 2014).

Desde el sector privado podemos destacar:

- Grandes espectáculos de flamenco en los teatros de las principales capitales de España, en ocasiones con más repercusión de la que podrían tener en Andalucía.

- Las peñas flamencas, en general pequeños locales con actividades flamencas semanales o mensuales en sus propias sedes y que a veces organizan un festival anual. El público de estos espectáculos no suele ir más allá de los aficionados de la zona.

2 http://www.andalucia.org/es/flamenco/el-flamenco-en-madrid-la-copa-pavon-segunda-llave-de-oro-delcante/ 
- Pequeños negocios de hostelería que incluyen algunas muestras de flamenco más bien por fiesta y, cuyo objetivo principal, está en mantener una clientela local.

- Tablaos flamencos, son herencia de los antiguos cafés-cantantes, en este caso el espectáculo se ofrece principalmente al turismo, en muchas ocasionas los aficionados al flamenco desconfían de la calidad del mismo (Díaz y Zalagaz, 2014).

\section{EL FLAMENCO Y SU IMPLICACIÓN EN EL TURISMO CULTURAL}

El flamenco es uno de los principales activos de la industria cultural española (Aoyama, 2007; Molina, 2014), y en especial en la andaluza (Ortega, 2006; Aoyama, 2009) no sólo por la transmisión de conocimientos expresados a través del cante y el baile, en espectáculos, festivales o concursos, sino por los beneficios económicos que genera (Calado, 2007; González y Ramírez, 2008; Cantero y Hernández, 2009; Ruiz y Pérez, 2011), pues la industria del flamenco está formada por un amplio abanico de bienes y servicios agrupados en diferentes sectores, los cuales se complementan y entrelazan hasta configurar el mercado global del flamenco, donde se pueden distinguir nueve sectores unidos con el flamenco: el textil, calzado, complementos, instrumentos musicales, audiovisual, revistas y libros, espectáculos, docencia y turismo. Es en este último sector donde el flamenco es uno de los elementos por los que nuestro país resulta más conocido (especialmente en Japón) desde el punto de vista cultural, siendo una de las fortalezas del país a la hora de captar turismo internacional formando parte de los atractivos que atraen a un turismo cuya motivación es conocer las tradiciones y cultura del lugar que visitan.

Se puede hablar del flamenco en cifras destacando las ventas discográficas de flamenco, en 2009 ascendieron en Andalucía el 9.2\% sobre las ventas de música popular y que a nivel nacional representaron el $4,6 \%$, lo que supuso 8 mill. $€$ y 20 mill. $€$ respectivamente. Asimismo en Andalucía el 19,4\% de los conciertos populares fueron dedicados al flamenco y en España el 9,5\% (Ruiz y Pérez, 2011).

El mismo estudio referenciado en el párrafo anterior indica como en 2009 se recibieron 700.000 turistas de flamenco con un gasto de 550 mill. $€$. (Ver el gráfico 1) en el que además se puede apreciar el volumen de negocio del flamenco en el sector de la moda y de la artesanía. Estas cifras dejan de manifiesto que el sector turismo en la industria del flamenco tiene una importante representatividad.

Si se observan los gráficos 2 y 3 , se puede ver a nivel nacional, como el número de asistentes a espectáculos flamencos disminuye desde 2010, sin embargo, la recaudación por la asistencia a estos espectáculos tiene un grado de decrecimiento menor. También se puede destacar, el hecho de que el porcentaje de asistentes a espectáculos flamencos sobre el resto de los espectáculos populares, tiene una destacada subida en 2010 aunque en los años siguientes se produce un decrecimiento y, por otra parte, el porcentaje de recaudación de espectáculos flamencos sobre el resto de los espectáculos populares se mantiene casi constante a partir de 2011 (Cervera, 2014). 


\section{Gráfico 1 \\ VOLUMEN DE NEGOCIO \\ DE SECTORES FLAMENCOS (2009)}

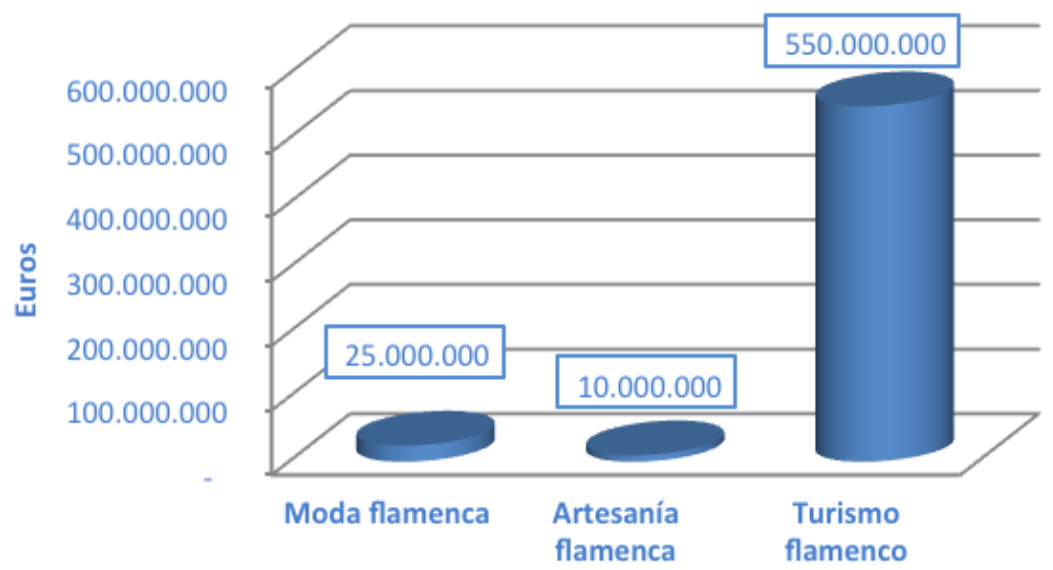

Fuente: elaboración propia, con datos obtenidos de Ruiz y Pérez (2011).

Gráfico 2

ASISTENCIA A CONCIERTOS FLAMENCOS

Y VALORACIÓN ECONÓMICA(€)

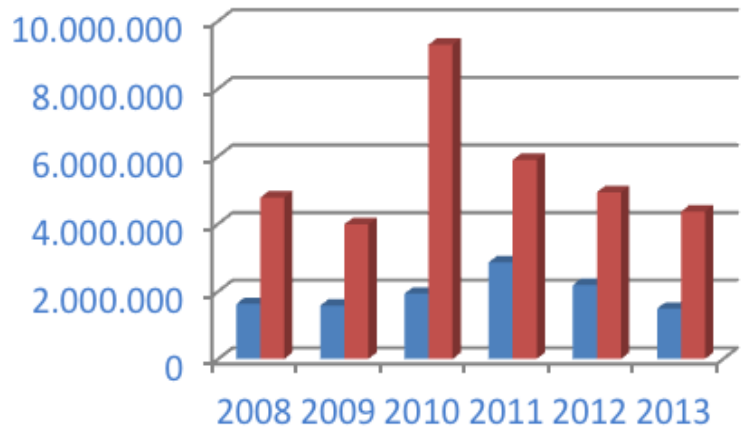

- Asistentes espectáculos flamencos

- Recaudación espectáculos flamencos

Fuente: elaboración propia a partir de datos de Informe SGAE 2014.

Ante este panorama negativo respecto a los ingreso del flamenco se podría ver recompensado con un aumento del turismo en dicha modalidad, especialmente el internacional, ofreciendo un producto cultural basado en el flamenco, como visitas a tablaos, museos del flamenco, etc. 


\section{Gráfico 3 \\ RELACIÓN ESPECTÁCULOS FLAMENCOS \\ SOBRE ESPECTÁCULOS POPULARES}

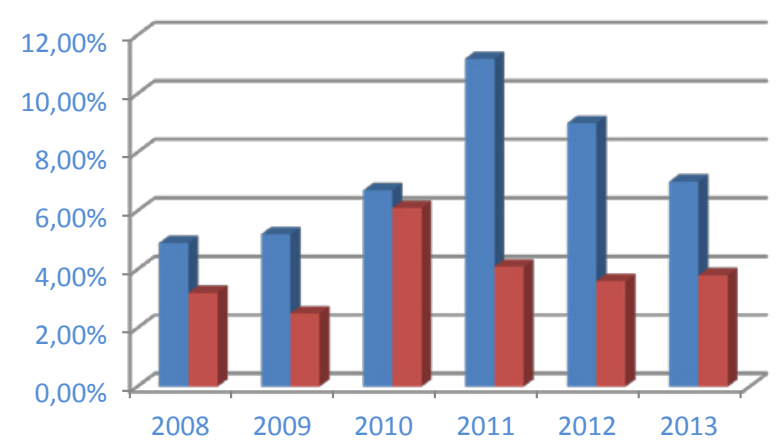

- \% asistentes flamencos sobre espectáculos populares

\% recaudación espectáculos flamencos sobre espectáculos populares

Fuente: elaboración propia a partir de datos de Informe SGAE 2014.

\section{ANÁLISIS DAFO DEL FLAMENCO COMO EXPRESIÓN DE TURISMO CULTURAL}

Son varios los factores que tienen influencia directa o indirecta sobre el desarrollo del flamenco como producto de turismo cultural, algunos son externos, que se expresan a través de amenazas o bien oportunidades, o en fortalezas y debilidades si estos factores son intrínsecos al flamenco como expresión artística o en su gestión empresarial. En consecuencia, el análisis DAFO que se presenta a continuación nos conduce a una apreciación de las potencialidades de esta modalidad turística y será el punto de partida para las propuestas estratégicas que se van a proponer.

\subsection{Debilidades}

Desde el punto de vista económico y empresarial una de las principales debilidades que se encuentran es la falta de regulación, esto da lugar a un volumen de economía sumergida, que por su propia definición es difícil de cuantificar. No es el caso de actividades organizadas por instituciones públicas o por organismos privados que reciben subvenciones de organismos públicos. Pero, son numerosos los eventos privados en los que actúan artistas de todos los niveles cuyas intervenciones no quedan reflejadas.

A nivel artístico, implica que en ocasiones se ofrecen espectáculos flamencos que no se ajustan a unos niveles mínimos de calidad, lo que da lugar no sólo al desprestigio de ese espectáculo sino del flamenco en general.

A pesar de que desde la Consejería de Cultura de la Junta de Andalucía, a través del Instituto Andaluz del Flamenco, se reconoce que el flamenco debe estar en los colegios, aún no ha llegado, los jóvenes avanzan en su formación cultural y musical en el desconocimiento de su propia cultura (Perales y Pedrosa, 2013, López, 2004). Podemos reconocer 
que algunos colegios o institutos incluyen algunas actividades extraescolares dedicadas al flamenco pero como algo muy marginal y sin reconocimiento oficial evaluable. A título de ejemplo, se puede indicar como el Ayuntamiento de Córdoba desde 2013 ha puesto en marcha un programa de difusión del flamenco en los centros escolares, consistente en que un porcentaje elevado de niños recibirán dos horas de formación con algunas nociones teórico prácticas.

Desde el punto de vista social, en origen, en muchas ocasiones se identificaron a los flamencos como «personas de mal vivir», este lastre hace que, aun hoy en día, parte de la sociedad más cercana a nuestra cultura, normalmente desde el desconocimiento, prefieren mantener cierta distancia, e incluso les preocupa que Andalucía y España se conozcan en el exterior por esta identidad artística.

\subsection{Fortalezas}

El flamenco es una marca de identidad, es un arte completamente diferente a cualquier otro, por su musicalidad, por su instrumentación y por su interpretación. Los «cantaores» tienen una fuerza en su expresión que, incluso, son capaces de llegar al corazón de personas que ni siquiera conocen el idioma. Para el turista con formación musical le resulta fácil admirar la expresión del flamenco a través de la guitarra, incluso mostrando un aprecio superior que los propios aficionados del lugar. Si nos centramos en el baile, que a su vez se funde con la guitarra, con el cante y a veces con otros instrumentos, se consigue un espectáculo no comparable a ninguna otra danza, la conjugación de manos, brazos y pies proporcionan un efecto explosivo que provoca grandes emociones entre los espectadores.

Los espectáculos de flamenco fuera de las fronteras españolas son cada vez más demandados; ya se ha comentado como el Instituto Andaluz del Flamenco apuesta por el desarrollo nacional e internacional. Proliferan los centros de enseñanza de flamenco en muchos países entre los que destacan, Japón, China, Estados Unidos... Algunas notificaciones de los informes de Extenda vienen a corroborar esta tendencia:

- «Extenda promociona el flamenco y la enseñanza del español en Japón con una misión comercial de empresas...»³.

- «La presente nota sectorial abordará el mundo del flamenco en China, su evolución en los últimos años, el estado actual y las perspectivas de futuro. El objetivo es hacer una aproximación al mundo del flamenco desde un punto de vista amplio, incorporando todo aquello relacionado con la moda flamenca y los instrumentos, los espectáculos y la enseñanza de este arte» ${ }^{4}$.

\footnotetext{
3 http://www.extenda.es/web/opencms/extenda/noticias/noticia_0748.html

4 http://www.extenda.es/web/opencms/fondo-documental/lectorFondo.jsp?uid=36e3de32-9f9a-11e38ba6-af00d39afa2c. El mundo del flamenco en China, 2013.
} 
El hecho de que se planteen estudios de flamenco oficiales con nivel de Grado en los Conservatorios Superiores de música, proporciona cimientos a este desarrollo musical. Es en el Conservatorio Superior de Música de Córdoba donde se puede obtener esta graduación en las siguientes modalidades:

- $\quad$ Especialidad Flamenco (Itinerario Cante flamenco).

- Especialidad Flamenco (Itinerario de Flamencología).

- $\quad$ Especialidad Flamenco (Itinerario de Guitarra flamenca).

En los últimos años se está produciendo un crecimiento del interés por el flamenco de los jóvenes, probablemente influenciados por artistas de la talla del cantaor Camarón de la Isla o del guitarrista Paco de Lucía. Esto se manifiesta en los múltiples concursos de jóvenes flamencos en los apartados de cante, baile y guitarra que se celebran en muchas ciudades andaluzas así como la existencia del Certamen Andaluz de Jóvenes Flamencos. Se trata de espectáculos que pueden ser de interés para el turismo pero que sin embargo no tienen suficiente difusión.

El sector público se muestra cada vez más involucrado con el apoyo al desarrollo del flamenco apoyando con medidas de formación y desarrollo, y organizando grandes espectáculos que son un referente para los amantes del flamenco nacionales e internacionales. Entre estos espectáculos podemos destacar:

- El festival Internacional de la Guitarra que se celebra en Córdoba anualmente, atrae a muchísimos expertos y aficionados de la guitarra de todo el mundo, como aficionados y como estudiantes de la música, (celebración anual desde 1981).

- La Noche Blanca del Flamenco en Córdoba busca una proyección para atraer al turismo interno y externo a base de tener una buena oferta y una buena difusión (evento anual desde 2008).

- La Bienal de Flamenco de Sevilla es un acontecimiento internacional del flamenco (nació en 1980).

- La Bienal de Flamenco de la Provincia de Málaga (en 2015 4ª edición).

- El Festival del Cante de la Minas de La Unión región de Murcia (primera edición en 1961).

- Festival de Flamenco de Jerez de la Frontera (Cádiz) organizado por el Ayuntamiento anualmente, se encuentra en su $19^{\text {a }}$ edición en 2015.

También es de destacar como negocios de hostelería complementan sus servicios con pequeñas muestras de espectáculos flamencos que en gran medida atraen al turismo. Proliferan los pequeños locales que casi a diario ofrecen pequeñas muestras de flamenco mientras el turista toma su cena o su copa.

\subsection{Oportunidades}

Muchos países europeos conceden a la formación musical un mayor protagonismo y por tanto una mayor formación musical entre sus habitantes, esto hace que la guitarra flamenca en modalidad solista tiene incluso mayor repercusión que dentro de España. 
En España se produjo un importante empuje a la formación musical a partir de 1992 con la creación de las Escuelas Municipales de Música, las cuales, en 2012 llegaron a alcanzar 265.000 alumnos en 280 escuelas públicas. Con los recortes a la cultura sólo en Madrid el número de estudiantes ha caído en un 40\%. En muchas de estas escuelas también se contempla la formación en flamenco. Algunos datos: «En Suecia, el 4\% de la población estudia música; en España, el 0,48\%» «En Alemania, los sistemas escolares difieren, pero todos ellos cuentan con asignaturas de música o, al menos, formación musical integrada en materias más amplias». En Suiza se aprobó la modificación de la Constitución para considerar la educación musical como un derecho básico (Verdú, 2012).

Las personas con una mayor formación musical son potenciales receptores del flamenco porque les resulta más fácil la comprensión del mismo. Esto es un elemento favorable al desarrollo del turismo cultural centrado en el flamenco.

En Japón existe una admiración máxima por el flamenco. Japón puede contar con ochenta mil personas que estudian en 650 academias repartidas por todo el país..$^{5} \mathrm{El}$ turismo japonés busca en España el flamenco, tanto desde el espectáculo como desde la formación. El japonés aficionado al flamenco suele ser practicante sobre todo en el baile. Es curioso como Atsuko Kamata, ganó el Premio Nacional en el Concurso Nacional de Córdoba en 1995 bailando por guajiras. Atsuko Kamata en la actualidad imparte clases de flamenco en su país.

El desarrollo de la formación en flamenco en Japón despierta un interés adicional por conocerlo en origen y por lo tanto es una oportunidad para nuestro turismo cultural.

Por el mismo motivo, aumentan las exportaciones de espectáculos flamencos, son muchos los artistas españoles que ofrecen sus servicios en el extranjero ofreciendo sus espectáculos y sus enseñanzas, lo cual facilita el flujo turístico hacia España y especialmente hacia Andalucía.

En un estudio realizado por la Junta de Andalucía sobre la demanda del turismo del flamenco se llegó a la conclusión en 2004 que el 3\% del turismo andaluz manifestó que su principal motivo para ir a Andalucía era el flamenco lo que representaba el $4 \%$ de los ingresos por turismo. El $60 \%$ de estos turistas procedía del extranjero, el $20 \%$ del resto de España principalmente Cataluña y Madrid y el resto de la propia Andalucía (Turismo Andaluz S. A., 2004). Aunque no está constatado se considera que estas cifras han ido evolucionando de manera favorable.

El acompañamiento instrumental en los espectáculos flamencos ha evolucionado, en origen se limitaba a la guitarra, palmas y taconeos en el baile en la actualidad se pueden encontrar instrumentos a ritmos flamencos que en origen sólo estaban vinculados principalmente con la música clásica, tales como la flauta travesera, el violín, el piano... (Troncoso,2011) esto abre al flamenco una oportunidad más, para relacionarse más de cerca con intérpretes de estos instrumentos o con aficionados a los mismos.

La nueva expresión musical del flamenco como flamenco fusión ofrece un género musical propio que aglutina el flamenco, el jazz (Zagalaz, 2013) y la música moderna en un nuevo estilo, modificando y adaptando sus dimensiones respectivas. Esto abre las puertas a la interpretación conjunta de grandes artistas de diferentes estilos musicales, los

5 https://www.flamenco-world.com/magazine/about/japon/ejapo15062004-2.htm 
más puristas del flamenco pueden ver este paso como una amenaza contra los cánones del flamenco. En realidad hay que verlo como la apertura del flamenco a otros estilos para engrandecerse conjuntamente. Podemos recordar a Camarón de la Isla junto a la Royal Filarmónica Orquesta de Londres en 1989. La fusión del flamenco con otros estilos musicales favorece el crecimiento de seguidores del flamenco (Blanco, 2014).

\subsection{Amenazas}

El hecho de que el flamenco es un arte con identidad, una expresión musical no comparable, una comunicación artística distante de cualquier otra aunque puedan darse composiciones de fusión, permite disponer de una oferta artística atrayente al turismo. Pero el aficionado al flamenco se desplazará donde encuentre los mejores espectáculos, donde sepa con seguridad que se van a encontrar el flamenco con alma, si ese espectáculo se ofrece y se identifica con Andalucía vendrá a Andalucía, pero si se ofrece o identifica con cualquier otro lugar se desplazará a ese lugar.

Por todo ello, es de considerar que asumir y gestionar la marca del flamenco como un valor propio y ofrecerlo como tal es muy importante, porque si desde otros lugares lo ofrecen como propio el turismo se desplazará a esos lugares.

En muchas ocasiones los espectáculos flamencos están gestionados por los propios artistas que a su vez no tienen formación en gestión de empresas, o por directivas de peñas que tampoco es su especialidad. Esto puede provocar la falta de sostenibilidad y escalabilidad de esta actividad empresarial cediendo espacio a otras actividades culturales mejor gestionadas.

Evidentemente la cultura es muy amplia y se entiende que hay que cuidar todos los estilos y modalidades, espectáculos de ópera, de música clásica, de jazz etc. son impresionantes pero si se quiere atraer al turismo estos espectáculos a Andalucía o a España no proporcionan identidad y probablemente el turista no se va a desplazar a Andalucía para verlos porque probablemente sea algo a lo que tiene acceso en su lugar de origen.

Los presupuestos de apoyo a la cultura se han reducido en los últimos años, desde 2009 hasta 2013 los presupuestos para empresas culturales se redujeron casi en un $50 \%{ }^{6}$

Según los informes nacionales del Observatorio de la Cultura, las disminuciones presupuestarias encadenadas en los últimos cinco ejercicios son las siguientes (Fundación Contemporánea, 2013):

- 2010 (respecto a 2009) - 21,3\%

- 2011 (respecto a 2010) - 17,6\%

- 2012 (respecto a 2011) - 18,9\%

- 2013 (respecto a 2012) - 15,1\%

- 2014 (respecto a 2013, previsión) - 5,9\%

Si el apoyo público a la cultura es cada vez menor las representaciones flamencas también quedarán perjudicadas.

6 http://economia.elpais.com/economia/2013/01/16/agencias/1358346057_160087.html 
Tabla 1

\section{MATRIZ DAFO - EL FLAMENCO Y EL TURISMO}

\section{Debilidades}

- Es una actividad no regulada en gran medida.

- No tiene un control de calidad de la oferta.

- Gran parte del sector opera dentro de la economía sumergida.

- Se ha creado una oferta de bajo nivel enfocada al turismo, que perjudica al flamenco y al turismo.

- No existe una formación reglada desde los colegios del flamenco.

- En origen no existe una valoración positiva generalizada del flamenco.

- No existe conciencia de marca.

- En España se sigue arrastrando cierta vergüenza de confesarse aficionado al flamenco.

- No existe una ruta del flamenco en las ciudades.

- El turista no puede contratar el espectáculo de flamenco en origen.

- No existen acuerdos con agencias de viajes o con webs de ventas de viajes que incorporen rutas flamencas.

- La falta de gestión empresarial.

Oportunidades

- En países del norte existe una mayor formación musical entre sus habitantes, por eso incluso la guitarra flamenca en solitario tiene una mayor repercusión.

- En Japón existe una admiración máxima por el flamenco. El turismo japonés busca en España el flamenco, tanto desde el espectáculo como desde la formación.

- La exportación del flamenco, genera turismo.

- La fusión del flamenco con otros estilos musicales.

- El reconocimiento de otros países hacia el flamenco como un gran arte.

\section{Fortalezas}

- Es una marca de identidad que no existe en ningún otro lugar.

- Es un elemento perfecto de atracción para el turismo, cuando se exporta fuera de nuestras fronteras tiene acogida máxima.

- Están implantados estudios oficiales de flamenco con nivel de licenciatura.

- Están surgiendo artistas jóvenes con una visión renovada del flamenco.

- El sector público está detectando una oportunidad en el desarrollo del flamenco como medio de posicionamiento en el sector turístico.

- El sector privado ve al flamenco como una oportunidad clara de rentabilidad.

- Desde 2010 el flamenco es Patrimonio Inmaterial de la Humanidad por la Unesco. 
Por otra parte, si las empresas que quieren financiar espectáculos flamencos no reciben una consideración fiscal especial por estas partidas no les interesa, les compensa más, por ejemplo, invertir en campañas de marketing que afectan a su cuenta de resultados reduciendo su base impositiva, y afecta directamente a su negocio. El propio Observatorio propone en su último informe la necesidad de desarrollar una ley de mecenazgo.

Por último, si la crisis continúa y se extiende a zonas de origen de nuestro turismo irremediablemente va a afectar a la oferta de arte flamenco.

\section{BUSINESS MODEL CANVAS APLICADO AL DESARROLLO DEL TURISMO DEL FLAMENCO}

Business Model Canvas es una herramienta que se utiliza para representar y sintetizar las características de los modelos de negocio, muy utilizada recientemente por los emprendedores para la creación de sus startups, pero también es aplicable para la modificación de modelos de negocio en marcha. (Osterwalder y Pigneur, 2011).

El Business Model es un lienzo que se divide en nueve módulos que se pueden agrupar en cuatro bloques que vienen a dar respuesta en relación a la empresa o proyecto a las siguientes cuestiones: ¿Quién?, ¿Qué?, ¿Cómo? y ¿Cuánto?

\section{Figura 5 \\ ESTRUCTURA DEL BUSINESS MODEL CANVAS}

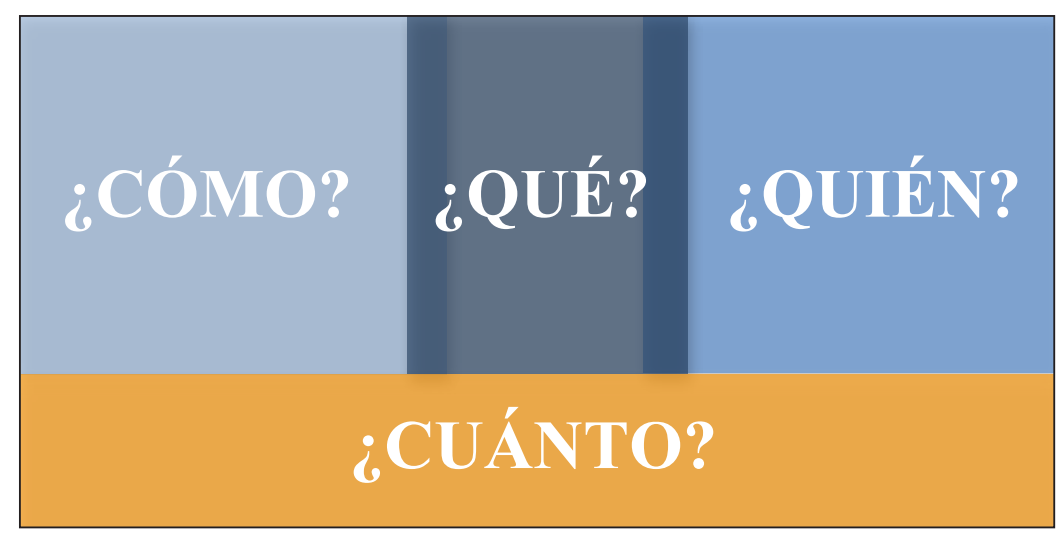

Fuente: elaboración propia a partir de Osterwalder y Pigneur, 2011.

Se trata de mostrar de forma esquemática a quién se dirige el proyecto, cuál es el perfil de los posibles destinatarios, cuál es el valor que aporta a dichos destinatarios, cómo se puede llevar este valor a los destinatarios y por último cómo se cuantifica este proceso desde el punto de vista del coste y de los ingresos.

El objetivo es que el turismo interesado por el flamenco visite Andalucía y encuentre de manera gratificante el producto que viene buscando. 
Se podría calificar de atrevimiento aplicar la herramienta Business Model Canvas para definir el turismo de flamenco como un todo. Para lo cual se van a describir los nueve módulos que componen el lienzo:

\section{Figura 6 \\ DETALLE DE LOS NUEVE MÓDULOS DE BUSINESS MODEL CANVAS}

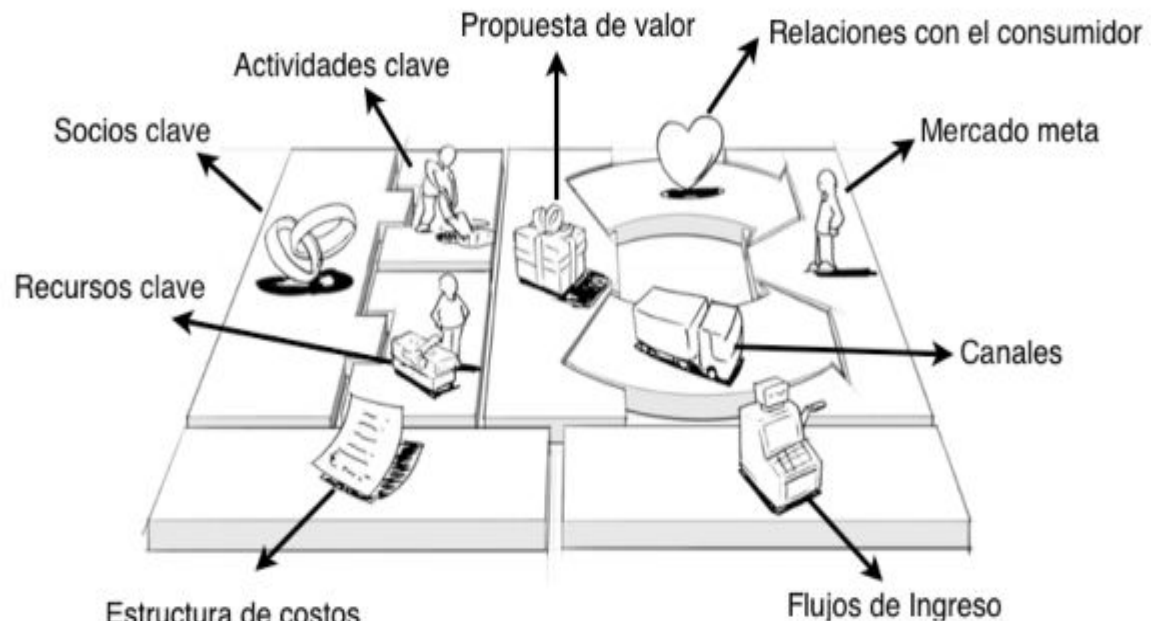

Estructura de costos

Fuente: http://www.deotramanera.co/node/58/galeria

\subsection{Mercado Meta}

Se pueden apreciar dos segmentos de clientes:

- Turistas conocedores del arte flamenco y que se desplazan buscando eventos concretos.

- Turistas que visitan la zona con una atracción general pero que son susceptibles de ser invitados a eventos flamencos.

\subsection{Propuesta de valor}

La propuesta de valor está formada por una serie de aportaciones como pueden ser:

- Expresión artística exclusiva.

- Formar parte de dicha expresión cultural, a través de los sentimientos. 
- Participar de los sentimientos de dolor o de tristeza de los intérpretes así como de las alegrías en sus «actuaciones por fiesta».

- Formación en cultura flamenca.

- Garantía de calidad en las representaciones.

- Para el turismo aficionado se le aporta especialmente más conocimiento, para el turismo no aficionado se le aporta nuevas experiencias de un arte exclusivo.

\subsection{Canales}

Los canales de comunicación son fundamentales para definir las estrategias que permitan aumentar el volumen de turismo.

Por una parte, hay que contemplar los canales de difusión y por otra los canales de oferta.

En cuanto a los canales de difusión es fundamental definir un plan de marketing, pero no se está hablando de una empresa concreta, sino de un conjunto de entidades públicas y privadas que en la actualidad se gestionan de forma independiente.

Existen web privadas que ofrecen la agenda de los espectáculos de flamenco que en muchas ocasiones se nutren de informaciones ocasionales que les van llegando. También se encuentran web de establecimientos que anuncian sus propios espectáculos.

Atendiendo a las grandes representaciones de reconocido prestigio éstas suelen tener asociado un plan de marketing capaz de alcanzar grandes distancias.

Se proponen los siguientes canales de difusión pero sería importante hacer un esfuerzo de unificación y coordinación:

- Redes sociales.

- Web especializadas.

- Agencias de viajes, muy importante para el turismo que quiere programar su viaje en torno al flamenco.

- Hoteles, muy importante para ofrecer al turismo indeciso la oferta de cada zona.

- Oficinas de turismo.

La oferta se sitúa en:

- Teatros.

- Espacios públicos de prestigio cultural, como pueden ser los patios de Córdoba.

- Peñas flamencas.

- Locales privados.

\subsection{Relación con el turismo}

Para el segmento aficionado al flamenco la relación puede ser online principalmente.

Para el segmento turismo cultural general es muy importante la relación personal a través de agencias, hoteles y oficinas de turismo que faciliten y propicien la motivación. 


\subsection{Ingresos}

En los locales privados, los ingresos provienen de las entradas y en muchos casos asociados a consumiciones en el local.

En estos locales, el turismo no tiene seguridad del nivel del espectáculo, sería conveniente establecer certificados de calidad donde se valoren, saber si se trata de artistas noveles o artistas experimentados con o sin relevancia en el mundo artístico.

En otras ocasiones, el espectáculo flamenco se ofrece como un complemento al servicio del local sin coste adicional para el cliente, sin ingreso por entrada. Esto ha dado lugar, en ciertos hoteles, a representaciones para turistas no experimentados con niveles inaceptables desde el punto de vista del arte flamenco.

Si se trata de teatros mantienen sus precios estipulados en función del nivel del evento organizado.

También existen espectáculos de flamenco organizados por entidades públicas que son de libre acceso, por lo tanto, no afectan directamente a los ingresos de la zona aunque indirectamente atraen al turismo aumentando los ingresos de restaurantes, comercios y hoteles.

Existen subvenciones destinadas explícitamente a actividades relacionadas con el flamenco.

Ingresos procedentes del mecenazgo, el hecho de que recientemente las deducciones fiscales hayan mejorado para los mecenas favorece la obtención de recursos, tanto de mecenas particulares como de empresas.

\subsection{Actividades clave}

Consideramos que hay una serie de actividades claves para mejorar el sector:

- Coordinación de actividades públicas y privadas.

- Regularización y valoración de los espectáculos con controles de calidad.

- Mantener la formación reglada en las distintas modalidades del flamenco que darán garantía a una oferta de nivel.

- Potenciar los planes de marketing.

\subsection{Recursos clave}

Los recursos clave hacen referencia a aquellos requerimientos sin los cuales el sector no saldría adelante.

Son recursos clave las personas que hacen posible la oferta cultural:

- Los artistas.

- Todas las que están al frente de cada uno de los eventos.

- Responsables de cultura en Ayuntamientos y Comunidades Autónomas.

- Responsables de peñas flamencas.

Lógicamente los recursos económicos son fundamentales, es conveniente el refuerzo en dotación pública y privada. 
También son recursos clave los recursos tecnológicos que potencien la difusión y la coordinación de los eventos.

\subsection{Alianzas clave}

Las alianzas claves ponen de manifiesto a entidades físicas o jurídicas con las que interesa mantener una relación con la cual se potencia el proyecto.

Pueden ser interesantes las alianzas con agencias de viaje y con las empresas de hostelería que facilitan la difusión y la ejecución.

Tanto en un caso como en otro, si estas empresas mantienen una oferta de espectáculos o rutas flamencas sería un complemento en el servicio a sus clientes, y al mismo tiempo sería un refuerzo para garantizar el éxito de los distintos eventos.

También son interesantes las empresas privadas que apuestan por la cultura patrocinando eventos flamencos, empresas que mantienen entre sus objetivos potenciar la cultura del flamenco.

A principios de 2015 se incorporan incentivos fiscales al mecenazgo, es decir, a personas físicas y jurídicas que aportan donaciones a proyectos de emprendedores, culturales, sociales o deportivos, que aunque no sea una medida específica para el flamenco sí puede servir para promover este arte. Aunque estas deducciones siguen estando por debajo de otros países europeos, tales como Francia, Italia o Reino Unido.

\subsection{Costes}

Los costes principales son:

- El coste de los artistas, aquí sería conveniente definir los cachés según categorías y evitar la situación actual en la cual hay artistas a los que se les asigna gratificaciones en muchos casos no declaradas muy por debajo de un valor estándar, y por otra parte a veces se aplican asignaciones procedentes de recursos públicos muy por encima de lo que ciertos artistas reciben habitualmente.

- El coste del montaje del espectáculo.

- El coste de difusión.

- El coste impositivo.

En referencia al coste impositivo tanto los artistas como los espectáculos de flamenco están sujetos a los tipos impositivos generales. Si se trata de personas físicas tienen un tratamiento similar al de cualquier profesional. Si se trata de personas jurídicas tampoco existe ninguna diferenciación. Teniendo en cuenta que el IVA pasó de un 10 a un $21 \%$ lo cual ha influido negativamente sobre todas las actividades culturales.

En la siguiente figura se muestra la representación esquemática del Business Model Canvas aplicado al turismo y el flamenco. Cada uno de los módulos podría ser susceptible de un estudio más profundo y pormenorizado que formará parte de próximos trabajos de investigación. 


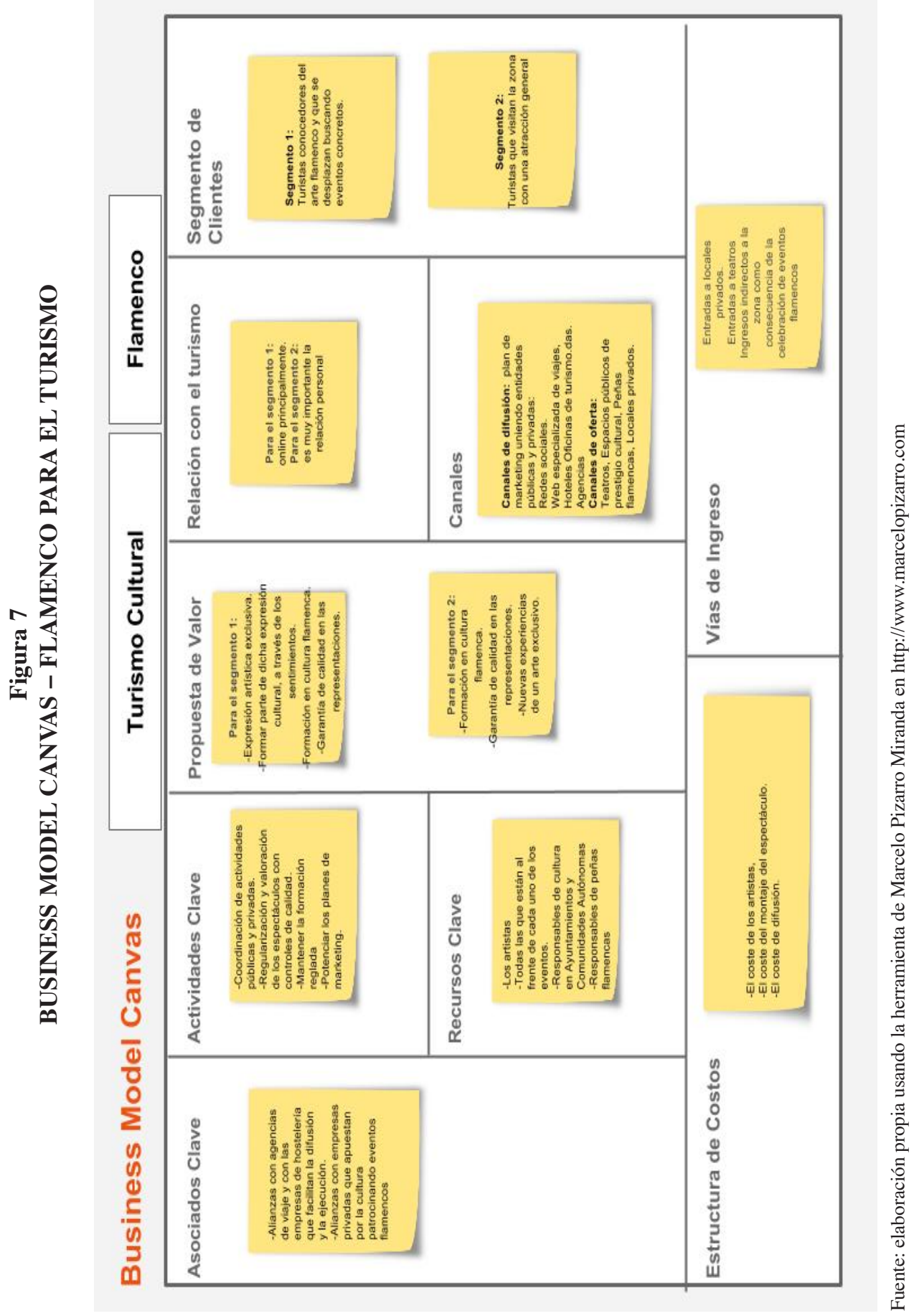




\section{CONCLUSIONES}

El flamenco es una marca de Andalucía y por extensión del resto de España. Es una expresión del arte en tres modalidades: cante, baile y guitarra, no comparable a ninguna otra expresión artística, por lo que fue declarado Patrimonio Inmaterial de la Humanidad por la UNESCO en el año 2010. Esta declaratoria dio, aún más si cabe, visibilidad a una expresión cultural propia que ya tenía demanda de turismo tanto fuera como dentro de España. Sin embargo, no existe todavía una visión del flamenco como producto de turismo cultural. Es, por ello, necesario plantear una estrategia de oferta rentable y escalable para el turismo del flamenco.

Se ha aplicado el Business Model Canvas al flamenco como valor que se ofrece a un segmento demandante que es el turismo. Una herramienta que se utiliza de forma estándar para representar el modelo de negocio de nuevas empresas o de nuevas líneas de negocio. Con esta herramienta se ha conseguido esquematizar los aspectos más relevantes del flamenco y el turismo visto como un todo desde la perspectiva de la gestión de empresa o de negocio.

Son muchos los factores a tener en cuenta en este desarrollo, algunos intrínsecos al mundo del flamenco y otros muchos externos, principalmente en el ámbito de las administraciones públicas. Así, se constata la necesidad de coordinación de las entidades públicas en la organización de eventos y en el desarrollo de estrategias de fomento, así como la necesidad de desarrollar un sistema de control de calidad que garantice al turismo eventos que cubran sus expectativas, o bien, que si parten del desconocimiento, no se marchen con una idea equivocada. Se necesita afianzar la formación en flamenco para que los artistas puedan ser, cada vez más, garantía de calidad.

La regularización del sector y la eliminación de la economía sumergida con definición de retribuciones según valía, potenciará tener una oferta más adaptada al desarrollo del sector turismo. Sería conveniente favorecer fiscalmente a las empresas que patrocinan espectáculos flamencos, aumentaría la oferta y proporcionaría rentabilidad indirecta. También favorecerá el turismo del flamenco el crear alianzas con empresas del sector turismo que faciliten la difusión y cuiden de la calidad, en su caso, de sus propias ofertas. Por último, es conveniente la realización de un plan de marketing capaz de llegar al turismo aficionado al flamenco para mantener su afición y su admiración, y capaz de atraer a ese otro turismo, todavía no aficionado, para que se despierte la curiosidad por conocer un arte con tanta identidad.

\section{BIBLIOGRAFÍA}

AGENDA DE LA EMPRESA (2014): «El flamenco Patrimonio andaluz al mundo». Agenda de la empresa andaluza: ideas, personas e instrumentos para dirigir la empresa, $\mathrm{n}^{\circ} 188$, pp. 62-64.

AOYAMA, Y. (2007): «The role of consumption and globalization in a cultural industry: The case of flamenco», Geoforum, n 38 , pp. 103-113.

AOYAMA, Y. (2009): «Artists, Tourists, and the State: Cultural Tourism and the Flamenco Industry in Andalusia, Spain», International Journal of Urban and Regional Research, vol. 33 (1), pp. 80-104. 
BLANCO, N. (2014): «ELOI CALDEIRO músico: «Todas las músicas son susceptibles de poder fusionarse»», Cambio 16, $\mathrm{n}^{\circ} 2.186$, pp. 54-55.

CÁCERES, R. y CAMPO, A. (2013): «Herreros y Cantaores: El trabajo de los metales en la génesis del flamenco», Revista de dialectología y tradiciones populares, Tomo 68, Cuaderno 2, pp. 445-467.

CALADO, S. (2007): El negocio del Flamenco. Sevilla, Signatura Ediciones de Andalucía.

CANTERO, J. y HERNÁNDEZ, E. (2009): La economía sevillana del flamenco. Cámara de Comercio de Sevilla. Sevilla, Confederación de Empresarios de Sevilla.

CARRASCO, M.A. (2014): «El flamenco en la cultura y la economía de Andalucía». Agenda de la empresa andaluza: ideas, personas e instrumentos para dirigir la empresa, $\mathrm{n}^{\circ} 188$, pp. 65.

CERVERA, R. (2014): «¿De qué hablamos cuando hablamos de música popular?». en Anuario SGAE 2014 de las artes escénicas, musicales y audiovisuales. Madrid, Fundación SGAE.

CRUCES, C. (2002): Historia del flamenco: siglo XXI. Sevilla, Tartessos.

CRUCES,C. (2014): «El flamenco como constructo patrimonial. Representaciones sociales y aproximaciones metodológicas». PASOS. Revista de Turismo y Patrimonio Cultural, vol. 12, nº 4, pp. 819-835.

DÍAZ, A.M. y ZALAGAZ, J. (2014): «Flamenco para aprender y convivir mejor». Cuadernos de Pedagogía, ${ }^{\circ}$ 444, pp. 25-28.

FUNDACIÓN CONTEMPORÁNEA, (2013): Observatorio de la Cultura, segundo semestre 2013, análisis y resultados.http://www.fundacioncontemporanea.com/pdf/ ANALISIS_RESULTADOS_OBS_DICIEMBRE_2013.pdf

GAMBOA, J.M. (2005):Una historia del flamenco. Barcelona, Espasa Calpe.

GÓMEZ, A. (2003): Cantes y estilos del flamenco. Córdoba, Universidad de Córdoba.

GONZÁLEZ, A. y RAMÍREZ, J. (2008): Impacto económico del XI Festival de Flamenco de Jerez. Cádiz, Fundación Provincial de la Cultura. Diputación de Cádiz.

HOLGUÍN, S. (2013): « «Vergüenza y ludibrio de las ciudades modernas»: los nacionalistas catalanes ante el flamenco en la Barcelona de 1900-1936», Hispania: Revista Española de Historia, vol. 73, n 244, pp. 439-468.

LÓPEZ, M. (2004): Introducción al flamenco en el currículum escolar. Málaga, Universidad Internacional de Andalucía.

MOLINAR, C.M.A. (2006): «Relaciones entre el turismo y la cultura: turismo cultural y cultura turística en México y en Colima», Estudios sobre las Culturas Contemporáneas, vol. XII, $\mathrm{n}^{\circ} 24$, pp. 9-23.

MOLINA, J.A.M. (2014):Relación entre el turismo y el crecimiento económico en España: la economía del flamenco. Sevilla, Universidad de Sevilla.

ORTEGA, C. (2006): El impacto del flamenco en las industrias culturales Andaluzas (Tesina). Madrid, Universidad Complutense de Madrid.

OSTERWALDER, A. y PIGNEUR Y. (2011): Generación de Modelos de Negocio, Deusto S. A. Ediciones.

PERALES, R. M. y PEDROSA, B. (2013): «Cambiemos la escuela: el arte flamenco en educación infantil y primaria», Creatividad y sociedad: revista de la Asociación para la Creatividad, $\mathrm{n}^{\circ} 21, \mathrm{pp} .1-23$ 
RUIZ, J. y PÉREZ, A. (2011): Estudio económico sobre la importancia del flamenco en las industrias culturales de Andalucía. Sevilla, Instituto Andaluz del Flamenco, Consejería de Cultura.

TORRES, N. (2014): «Guitarra popular rasgueada «pre-flamenca» en la primera mitad del siglo XIX: Fuentes escritas», Revista de investigación sobre Flamenco: La Madrugá, $\mathrm{n}^{\circ} 10$, pp. 55-120.

TOSELLI, C. (2003): «Turismo cultural, participación local y sustentabilidad. Algunas consideraciones sobre la puesta en valor del patrimonio rural como recurso turístico en Argentina». Estudio Compartido sobre Turismo y Cultura publicado en el Portal Iberoamericano de Gestión Cultural.

TROITIÑO, M.A. y TROITIÑO, L. (2009): «Turismo y patrimonio en Castilla y León: las ciudades patrimonio de la humanidad (Ávila, Salamanca y Segovia) como destinos turísticos de referencia», Polígonos. Revista de Geografía, no 19, pp. 145-178.

TRONCOSO, J. (2011): El piano flamenco: génesis, recorrido diacrónico y análisis musicológico. Sevilla, Universidad de Sevilla.

TURISMO ANDALUZ S.A. (2004): La demanda de Turismo de Flamenco en Andalucía 2004. Sevilla, Consejería de Turismo, Comercio y Deporte.

VERDÚ, D. (2012):«España manda la música a otra parte» El País.

VERGILLOS, J. (2002): Conocer el flamenco: sus estilos, su historia. Sevilla, Signatura Ediciones.

ZAGALAZ, J. (2013): «El impacto del jazz en la renovación del flamenco: una perspectiva analítica a través de la obra de Paco de Lucía entre 1978 y 1981», en Musicología global, musicología local, pp. 863-880. 
